\title{
Residencias Geriátricas. Análisis del marco jurídico desde la perspectiva de la provincia del Chaco, Argentina
}

\author{
Geriatrics Residences. Legal framework analysis from \\ Argentina, Chaco province`s perspective
}

\section{Ayelén Anzola}

\section{RESUMEN}

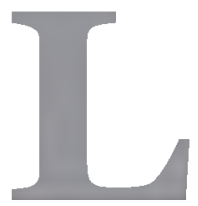

a realidad del adulto mayor presenta características que responden a los cambios internos propios de la biología de la persona y a cambios externos. Estos impactan en sus necesidades, las cuales a su vez se modifican a lo largo del tiempo. En este contexto, las residencias geriátricas como instituciones complejas se presentan como una solución frente a las contingencias de la vejez y la salud, cumpliendo una verdadera función social. Desde este punto de vista intentaremos responder si la ley, específicamente de la Provincia del Chaco, en Argentina contempla adecuadamente las necesidades de los adultos mayores.

Palabras clave: adultos mayores; marco jurídico de las residencias geriátricas; derecho local de la ancianidad; contingencias del adulto mayor.

\section{ABSTRACT}

The elderly's reality has characteristics that respond to the internal changes of the person's biology and external changes. These ones impact on their needs which in turn change over time. In this framework, geriatric residences as complex institutions are presented as a solution to the health and old age contingencies, fulfilling a true social function. From this point of view, we will try to respond if the law, specifically the Chaco Province regulation, adequately contemplates the needs of elderly.

Keywords: elderly; geriatric legal framework; elderly local law; old age contingencies.

COMO CITAR ESTE ARTÍCULO Ayelén Anzola. (2021). Residencias Geriátricas. Análisis del marco jurídico desde la perspectiva de la provincia del Chaco, Argentina. Revista de la Facultad de Ciencias Económicas, 26(1), $27-45$

http://dx.doi.org/10.30972/rfce.2615028

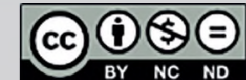

https://creativecommons.org/licenses/by-ne-nd/4.o/ Revista de la Facultad de Ciencias Económicas ISSN 1668-6357 (formato impreso) ISSN 1668-6365 (formato digital) por Facultad de Ciencias Económicas Universidad Nacional del Nordeste (UNNE) Argentina se distribuye bajo una Licencia Creative Common Atribución - No Comercial - Sin Obra Derivada 4 o Internacional. 


\title{
NUEVO PARADIGMA DE LA VEJEZ
}

\author{
"¿Cuánto vive el hombre, por fin? \\ ¿Vive mil días o uno solo? \\ ¿Una semana o varios siglos? \\ ¿Por cuánto tiempo muere el hombre? \\ ¿Qué quiere decir "Para siempre”"? \\ Pablo Neruda
}

El hombre naturalmente envejece cada día de su vida. El paso de los años provoca en su ser la ocurrencia de contingencias o el surgimiento de necesidades y cada una de ellas requiere diferentes respuestas. Así, se manifiestan los primeros cambios intrínsecos a la naturaleza misma del hombre.

Sin embargo, existen otros cambios, a los que podemos llamar externos y que también modifican o hacen nacer necesidades distintas de las que un grupo de personas pudo haber tenido con anterioridad. Este segundo grupo de factores interviene en las formas en las cuales se vive la vejez.

Esto es así ya que el envejecimiento de la población se desarrolla junto a otros cambios, en la convergencia de ambos se producen nuevos fenómenos que impactan en esta etapa de la vida de la persona humana.

Un ejemplo de ello pueden ser los cambios sociales; las modificaciones de jornadas laborales: aquellas personas que tienen jornadas de trabajo más extensas no pueden cuidar a los familiares que se encuentran transitando la etapa de adultos mayores; o los cambios tecnológicos: la rapidez con la que introducimos nuevas tecnologías muchas veces provoca que el adulto mayor no logre adaptarse a este ritmo de cambio y necesite asistencia para llevar adelante tramites muy simples, como percibir su jubilación o presentar alguna documentación ante la obra social; agrego a esto, que quizás el efecto más perjudicial en esta dificultad de adaptación es que, ésta se convierte en una suerte de aislamiento del adulto mayor en la sociedad.

Estas necesidades, ya sean intrínsecas o extrínsecas, no siguen obligatoriamente un patrón ni permanecen iguales a lo largo del tiempo. No obstante, a través de las estadísticas, podemos extraer algunos datos duros que nos permiten entender cuál es el sentido en el que cambian estas necesidades y encontrar a estos problemas respuestas integrales.

En este sentido, el sostenimiento de la expectativa de vida ha demostrado algunas características que redefinen la vejez. En Argentina, la esperanza de vida ha crecido notablemente. 
Cuadro 1: Porcentaje de la población de 65 años y más y edad mediana de la población por sexo. Total del país. Período 2010-2040.

\begin{tabular}{|c|c|c|c|c|}
\hline \multirow{2}{*}{ Año } & \multicolumn{2}{|c|}{$\begin{array}{c}\text { Porcetanje de población de } 65 \\
\text { años y más }\end{array}$} & \multicolumn{2}{|c|}{$\begin{array}{l}\text { Edad } \\
\text { mediana }\end{array}$} \\
\hline & Varones & Mujeres & Varones & Mujeres \\
\hline & & & \multicolumn{2}{|c|}{ Años } \\
\hline 2010 & 8,5 & 11,7 & 28,4 & 30,7 \\
\hline 2025 & 10,5 & 14,2 & 31,5 & 34,2 \\
\hline 2040 & 13,1 & 17,4 & 35,1 & 38,2 \\
\hline
\end{tabular}

Fuente: INDEC. Estimaciones y proyecciones elaboradas en base a resultados del Censo Nacional de Población, Hogares y Viviendas 2010.

Si observamos los datos obtenidos en el censo del año $2010^{1}$ por el Instituto Nacional de Estadísticas y Censos, vemos que en el año 1960 el porcentaje de personas mayores de 60 años era de un 5,5\% respecto al total de habitantes en ese mismo año. Sin embargo, en el año 2010 la población adulta mayor a 60 años alcanzó el 10,2\% del total de la población y la expectativa para los próximos años es el aumento de estos porcentajes. Es decir que, con los años Argentina tendrá una población más envejecida. Este es el primer dato poblacional que debemos tener en cuenta cuando analizamos el colectivo de adultos mayores.

Cuadro 2: Estructura de la población por sexo y grupo quinquenal de edad.Total del país. Año 2010

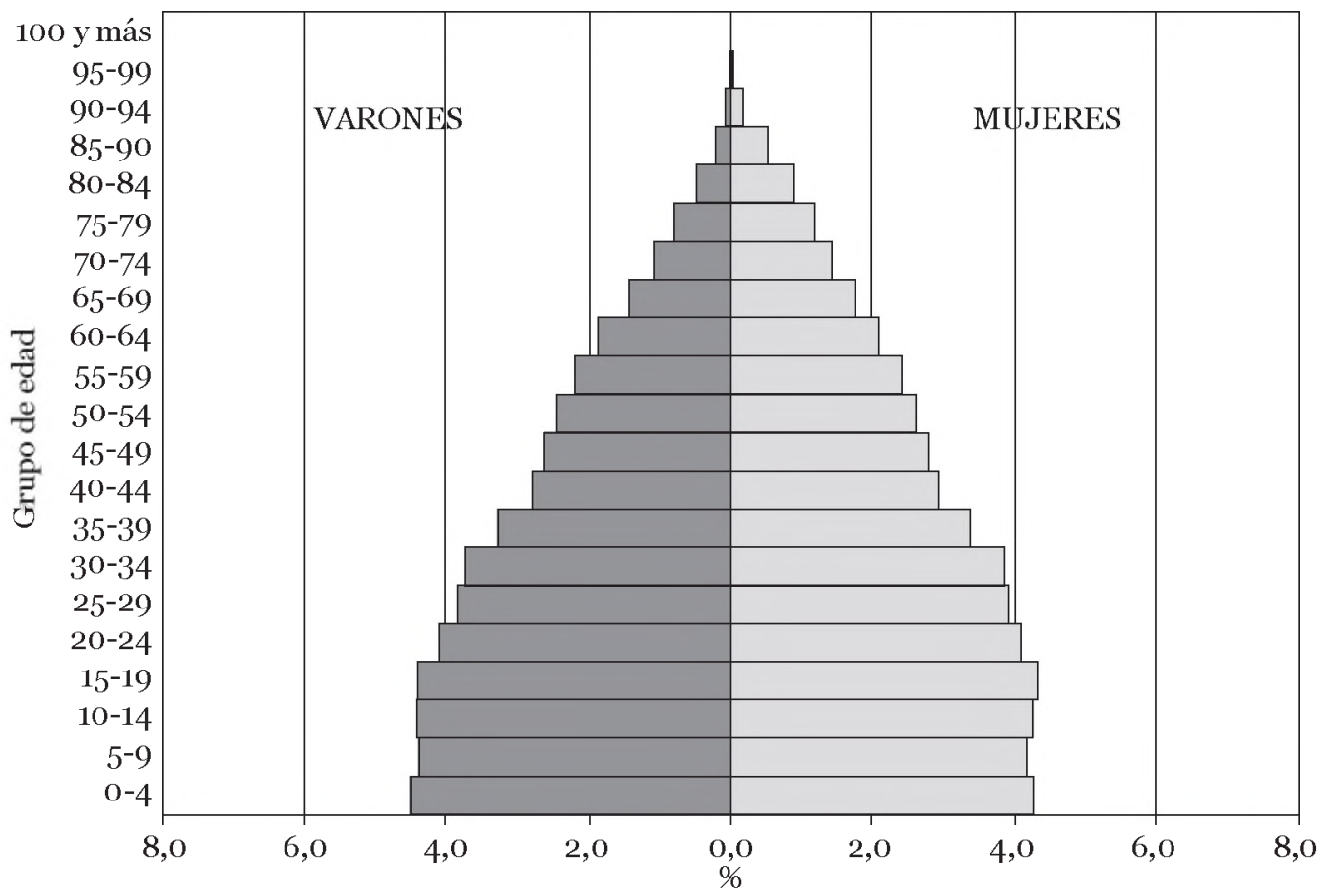

Fuente: INDEC. Estimaciones y proyecciones elaboradas en base a resultados del Censo Nacional de Población, Hogares y Viviendas 2010.

${ }^{1}$ INDEC. (2010). Estimaciones y proyecciones de población 2010-2040 - Total del País - Serie análisis demográfico (35). Recuperado de www.indec.gob.ar 
Cuadro 3: Estructura de la población por sexo y grupo quinquenal de edad. Total del país. Año 2025

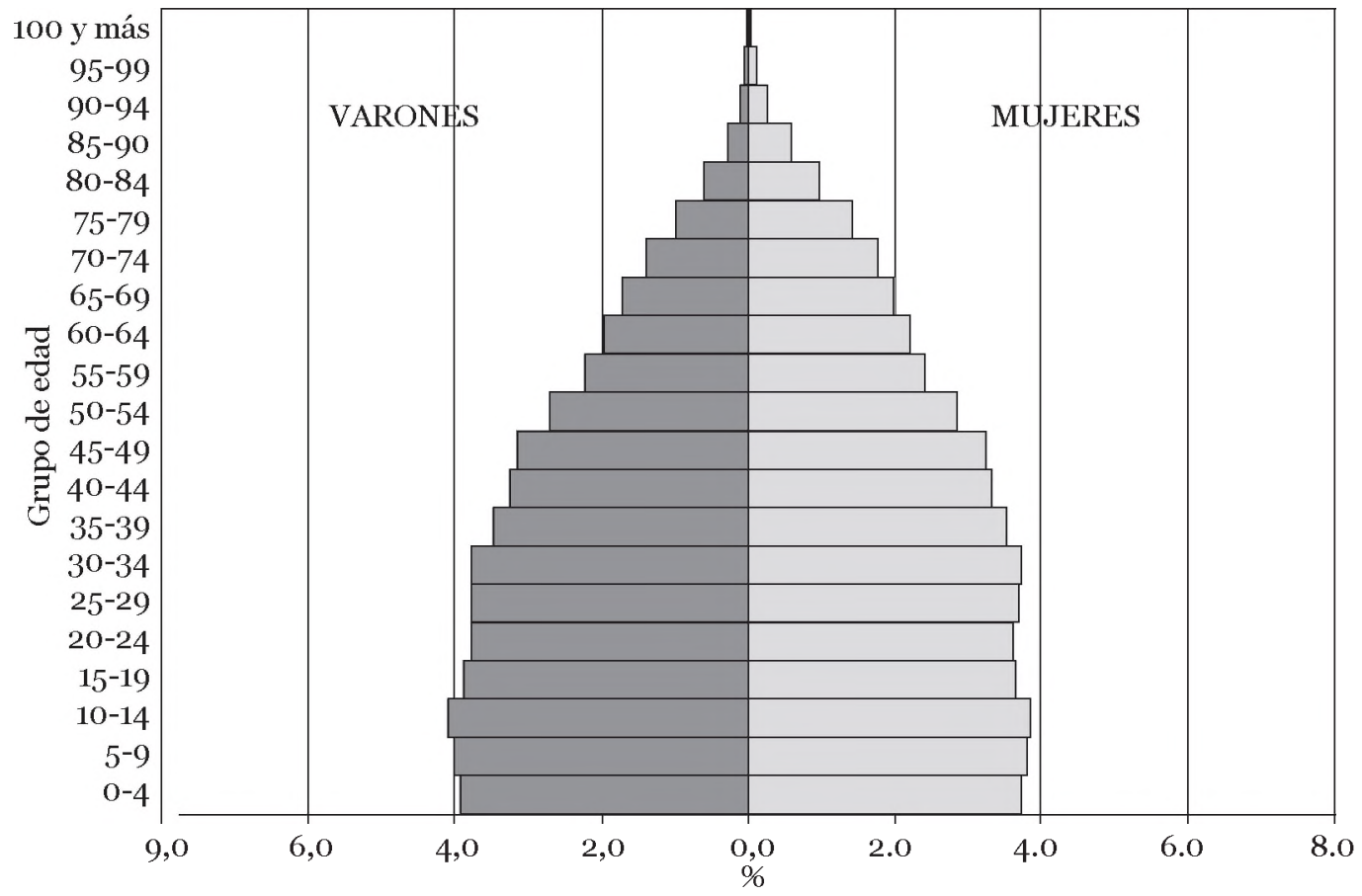

Fuente: INDEC. Estimaciones y proyecciones elaboradas en base a resultados del Censo Nacional de Población, Hogares y Viviendas 2010.

Otra de las características que encontramos relevantes dentro de estos datos es que no solo observamos una población más longeva, sino que dentro de este grupo existe una población femenina mayor que la masculina. También observamos que la vejez se prolonga en una duración de 20 años o más. Como resulta lógico, esto conlleva la coexistencia de dos o más generaciones envejecidas. 
Cuadro 4: Estructura de la población por sexo y grupo quinquenal de edad. Total del país. Año 2040

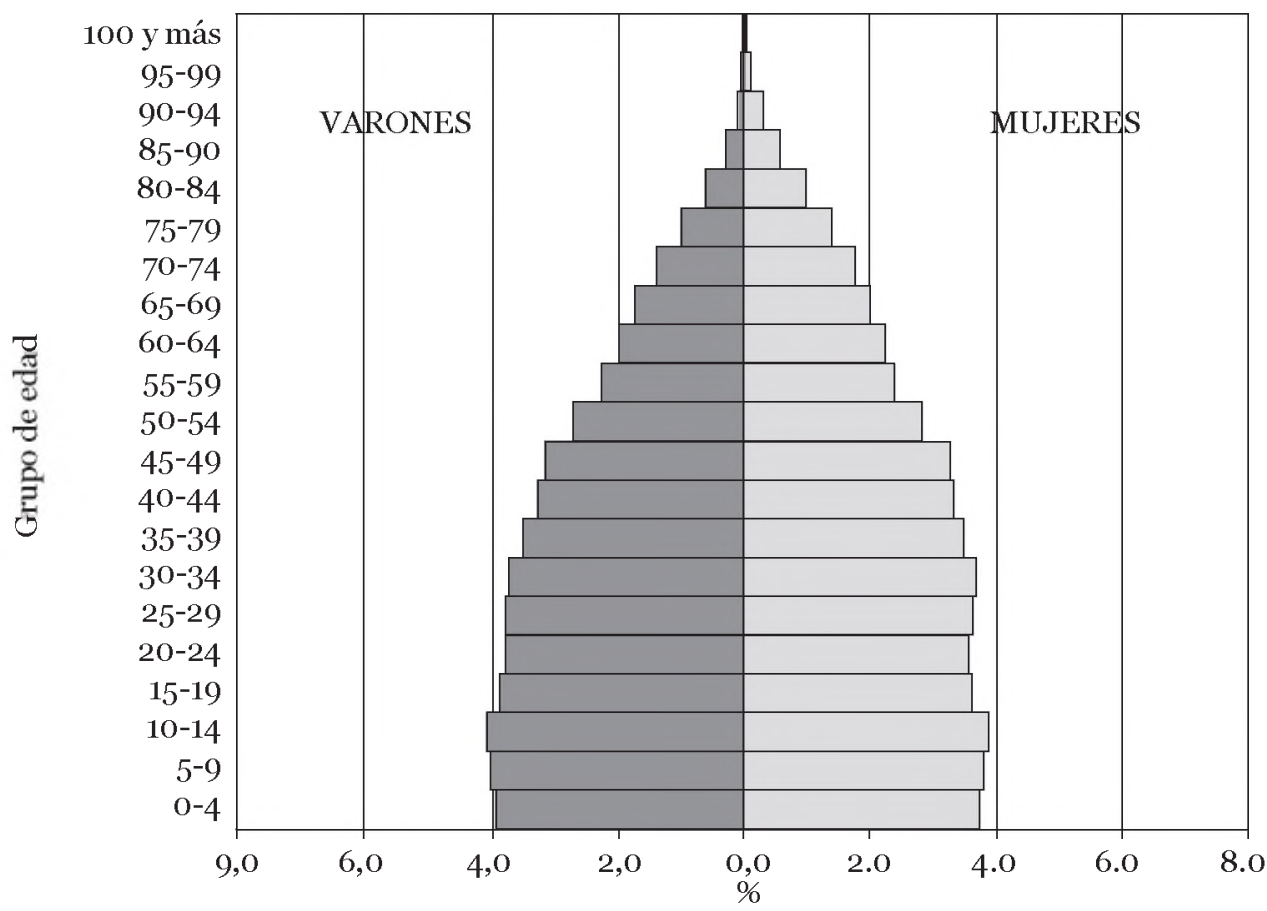

Fuente: INDEC. Estimaciones y proyecciones elaboradas en base a resultados del Censo Nacional de Población, Hogares y Viviendas 2010.

En palabras de Dabove se da en nuestra sociedad:

"La existencia simultánea y en expansión de dos generaciones envejecidas, no siempre vinculadas por lazos de parentesco, junto a la coexistencia de tres o cuatro generaciones partícipes de una misma familia: bisabuelos, abuelos, padres e hijos; a la convivencia de dos generaciones sucesivas de personas envejecidas y vinculadas por lazos de familia" (Dabove, 2008, pp. 39-54).

Tal como expresa la autora precitada (2016), el envejecimiento, desde el punto de vista cultural, se caracteriza porque en él convergen distintas generaciones que expresan a su vez distintas formas de comprender la vida, diferentes opiniones políticas que responden a los distintos momentos históricos en los que cada persona ha vivido, valores y experiencias. Es importante resaltar esta idea porque, a mi entender, según como se conjuguen estas subjetividades vamos a tener como resultado una sociedad inclusiva o excluyente del adulto mayor.

Aquellas personas nacidas en la generación de 1960 en Argentina tendrán seguramente una visión distinta de la historia respecto de aquellos nacidos después del año 1983, año en que retornó la vida democrática, pudiendo corresponder sin dudas a una generación de padre e hijo, madre e hija. Lo mismo ocurriría si pensáramos, como en el ejemplo antes dado, en el uso de aparatos digitales tales como la computadora, un dispositivo móvil como el ce- 
lular, uso de internet y herramientas digitales, etc. Cada una de estas generaciones convive. Por este motivo, existe una relación innegable entre la juventud y la vejez.

Como hemos dicho, una población envejecida multigeneracional presenta problemas propios. En muchos casos los hijos, también mayores de 60 años se ven afectados por contingencias físicas y económicas que les impide ayudar a sus padres y, los nietos o hijos menores de 60 años, se encuentran en etapa laboral por lo cual el tiempo que disponen para su cuidado o atención es muy limitado.

En línea con lo antes dicho, debemos comprender que el envejecimiento de la población es una realidad de toda la sociedad. Por lo tanto, asegurar una vejez en condiciones dignas importa también asegurar a la juventud una vida digna.

\section{RESIDENCIAS GERIÁTRICAS}

\section{FUNCIÓN SOCIAL}

Las residencias geriátricas o gerontológicas son instituciones que surgen como respuesta de una realidad social específica que es: dar respuesta a las necesidades de alojamiento y cuidados de la población que ya ha alcanzado la vejez.

La idea del adulto relegado del contexto social en el que se halla inserto resulta inadmisible $y$, en consecuencia, los geriátricos aparecen como instituciones que cumplen una verdadera función social. Una función o sistema supone un conjunto de piezas que interactúan entre sí para lograr un objetivo común. Las residencias o instituciones geriátricas asumen, en este sentido, la protección de un aspecto de la vida del ser humano, sin el cual, la dignidad de la persona no se halla completa. Son, en otras palabras, instituciones que velan por los derechos humanos en un estadio determinado de la vida.

Chirinos (2011) ha dicho que "La seguridad social es el signo visible del verdadero sentir de la sociedad frente a las necesidades, y la extensión de las prestaciones también son signos de la posición social frente a los valores que inspiran el accionar de las autoridades" (Chirinos, 2011, p.3). Asegurar esos valores también significa que la previsión social no es ajena a la realidad que enfrentan las sociedades.

Las residencias geriátricas responden a una necesidad habitacional sin dudas, pero no podemos olvidar que también responden a las características específicas del adulto mayor. Su estructura responde a las exigencias de justicia individualizada y tiene, por fin último, lograr el fortalecimiento de las personas mayores en tanto sujetos vulnerables del sistema social, político y jurídico.

Ahora bien, dicho esto, no podemos perder de vista que la función de las residencias geriátricas en la sociedad puede ser analizada desde distintos aspectos. 
Según el sujeto al que está dirigido, sin dudas el adulto mayor aparece como primer beneficiario, pero no es el único. No debemos perder de vista que todos somos adultos mayores en potencia, $y$, por lo tanto, tarde o temprano podríamos estar interesados en los servicios que la geriatría nos brinda. Quizás desde una perspectiva un tanto egoísta, asegurar la prestación de este servicio importa a la juventud como sistema de protección para una etapa futura en su vida.

Según las contingencias o necesidades a las que responde, vemos que las instituciones geriátricas tienen a su cargo la protección de la contingencia biológica de la vejez y las potenciales contingencias patológicas que afecten la salud. Sin duda el factor de la edad incide en la salud de las personas. A mayor edad es menor la reproducción celular del ser humano. Esta verdad absoluta desde el punto de vista de la biología nos lleva a suponer que un adulto mayor se recuperará más lentamente que una persona de menor edad que atraviese su misma enfermedad.

Esta doble protección convierte a los geriátricos en instituciones complejas, ya que dentro de las particularidades habitacionales que debe prever, se encuentran las contingencias patológicas que también se han puesto a su cargo. Este es, posiblemente uno de los puntos de mayor conflicto desde el punto de vista de su encuadre legal y el reconocimiento de las prestaciones que efectivamente brindan las instituciones geriátricas.

\section{NATURALEZA JURÍDICA}

De manera previa advertimos que la legislación aplicable a las residencias geriátricas es aún un trabajo en proceso en Argentina. Aunque a continuación pondremos bajo análisis las múltiples normas y ramas del derecho que contemplan sus relaciones jurídicas y funcionamiento, podremos observar que, por otro lado, todas ellas prescinden dentro de sus previsiones regulatorias de algún aspecto característico de las residencias geriátricas. Esta carencia vuelve deficiente la legislación aplicable y coloca nuevamente al adulto mayor en una posición de vulnerabilidad. Consideramos pertinente en este punto, exponer cada una de las estructuras previstas por el ordenamiento jurídico argentino y contrastarla con la realidad que exponemos.

Así las cosas, desde el punto de vista del derecho civil, y, tomando en consideración el vínculo que rige la relación entre el residente y la institución, podemos decir que es un contrato atípico o de hospedaje. Esta idea de relación jurídica contractual es, a mi modo de ver, deficiente porque únicamente tiene en cuenta las obligaciones bilaterales que se desarrollan en torno al hospedaje del adulto mayor.

Tomando en cuenta la finalidad de la institución, se ha dicho que el fin público corresponde a la persona pública y el fin privado corresponde a la persona privada (Mayer, 1954) y por lo tanto el imperium y el control administrativo se ejercen solo sobre las primeras. También 
corresponde a las primeras la prestación de un servicio que se califica como público ${ }^{2}$ y la satisfacción de intereses también públicos.

\section{LEGISLACIÓN APLICABLE}

La vejez concebida como una etapa o estadio de la vida ha atravesado a lo largo de la historia distintas realidades. El Derecho de la Vejez o de la Ancianidad surgió con la convención de Viena de 1982 que dio origen a la "Resolución de la Asamblea Mundial de Naciones Unidas sobre el envejecimiento" despertando desde el reconocimiento de la vejez a la voluntad de los estados para protegerla.

El primer antecedente en nuestro país de este marco normativo internacional se encuentra en la Convención Interamericana sobre la protección de los derechos humanos de las Personas Mayores, aprobada el 15 de junio de 2015.

El objeto de la Convención es "promover, proteger y asegurar el reconocimiento y el pleno goce y ejercicio, en condiciones de igualdad, de todos los derechos humanos y libertades fundamentales de la persona mayor, a fin de contribuir a su plena inclusión, integración y participación en la sociedad". Se establecen tres acciones positivas para que la persona mayor de 60 años goce plenamente de los derechos que le son propios. A renglón seguido, en su art. 2 establece la prohibición de discriminación, que junto con la declaración de igualdad mencionada en el art. 1 protege al adulto mayor contra la categorización o actos arbitrarios en desmedro de la persona. ${ }^{3}$

En particular, el art. 12 enuncia los derechos de la persona mayor que recibe servicios de cuidado a largo plazo. Este es un antecedente sin precedente en nuestra legislación. Se prevé un sistema integral de cuidados para la protección y promoción de la salud, cobertura de servicios sociales, seguridad alimentaria y nutricional, agua, vestuario y vivienda; promoviendo que la persona mayor pueda decidir permanecer en su hogar y mantener su independencia

\footnotetext{
${ }^{2}$ Dabove; M. I. (septiembre; 2013a). Elder law: a need that emerges in the course of life. Ageing International; 40; 138.148. doi 10.1007/ s12126-013-9193-4. Ha dicho la autora que las residencias geriátricas son instituciones "prestadoras de servicios sociales en función de su objeto o finalidad: aseguran la vivienda para los mayores de edad, promueven la salud pública, impulsan mejoras en la calidad de vida de la población en general, etc.".

${ }^{3}$ Art. 2: "Discriminación": Cualquier distinción, exclusión, restricción que tenga como objetivo o efecto anular o restringir el reconocimiento, goce o ejercicio en igualdad de condiciones de los derechos humanos y las libertades fundamentales en la esfera politica, económica, social, cultural o en cualquier otra esfera de la vida pública y privada.

"Discriminación múltiple": Cualquier distinción, exclusión o restricción hacia la persona mayor fundada en dos o más factores de discriminación.

"Discriminación por edad en la vejez": Cualquier distinción, exclusión o restricción basada en la edad que tenga como objetivo o efecto anular o restringir el reconocimiento, goce o ejercicio en igualdad de condiciones de los derechos humanos y libertades fundamentales en la esfera política, económica, social, cultural o en cualquier otra esfera de la vida pública y privada.

"Persona mayor que recibe servicios de cuidado a largo plazo": Aquella que reside temporalo permanentemente en un establecimiento regulado sea público, privado o mixto, en el que recibe servicios socio-sanitarios integrales de calidad, incluidas las residencias de larga estadía, que brindan estos servicios de atención por tiempo prolongado a la persona mayor, con dependencia moderada o severa que no pueda recibir cuidados en su domicilio.
} 
y autonomía. Esta enunciación pone de manifiesto la doble protección de las residencias geriátricas ante las contingencias. Por tanto, corresponde a estas instituciones cubrir las necesidades que resulten de la contingencia biológica de la vejez y las potenciales contingencias patológicas que afecten la salud del adulto mayor. ${ }^{4}$

Salud y vejez deben ser cuestiones que tratar en paralelo, pese a lo distintas que estas contingencias sean. Un tratamiento simultáneo de las mismas demuestra una correcta visión de la realidad. No podemos desconocer que el factor de la edad incide en la salud de las personas, porque a mayor edad es menor la reproducción celular del ser humano. Esta verdad absoluta del mundo de la biología nos lleva a suponer que un adulto mayor se recuperará más lentamente que una persona de menor edad que atraviese su misma enfermedad. También debemos considerar las enfermedades degenerativas de la salud que se presentan en las personas que superan los 60 años.

Esta doble protección convierte a los geriátricos en instituciones complejas que dentro de las particularidades habitacionales que debe prever, se encuentran las contingencias patológicas que también se han puesto a su cargo.

Este quizá es uno de los puntos de mayor conflicto desde el punto de vista de su encuadre legal y el reconocimiento de las prestaciones que efectivamente prestan las instituciones geriátricas, por lo cual se ha incluido un artículo especial en relación con la protección de la salud del adulto mayor.

\footnotetext{
${ }^{4}$ Artículo 19 -Derecho a la salud: La persona mayor tiene derecho a su salud física y mental, sin ningún tipo de discriminación. Los Estados Parte deberán diseñar e implementar políticas públicas intersectoriales de salud orientadas a una atención integral que incluya la promoción de la salud, la prevención y la atención de la enfermedad en todas las etapas, y la rehabilitación y los cuidados paliativos de la persona mayor a fin de propiciar el disfrute del más alto nivel de bienestar, físico, mental y social. Para hacer efectivo este derecho, los Estados Parte se comprometen a tomar las siguientes medidas: a) Asegurar la atención preferencial y el acceso universal, equitativo y oportuno en los servicios integrales de salud de calidad basados en la atención primaria, y aprovechar la medicina tradicional, alternativa y complementaria, de conformidad con la legislación nacional y con los usos y costumbres.

b) Formular, implementar, fortalecer y evaluar políticas públicas, planes y estrategias para fomentar un envejecimiento activo y saludable. c) Fomentar políticas públicas sobre salud sexual y reproductiva de la persona mayor. d) Fomentar, cuando corresponda, la cooperación internacional en cuanto al diseño de políticas públicas, planes, estrategias y legislación, y el intercambio de capacidades y recursos para ejecutar planes de salud para la persona mayor y su proceso de envejecimiento. e) Fortalecer las acciones de prevención a través de las autoridades de salud y la prevención de enfermedades, incluyendo la realización de cursos de educación, el conocimiento de las patologías y opinión informada de la persona mayor en el tratamiento de enfermedades crónicas y otros problemas de salud. f) Garantizar el acceso a beneficios y servicios de salud asequibles y de calidad para la persona mayor con enfermedades no transmisibles y transmisibles, incluidas aquellas por transmisión sexual. g) Fortalecer la implementación de políticas públicas orientadas a mejorar el estado nutricional de la persona mayor. h) Promover el desarrollo de servicios socio-sanitarios integrados especializados para atender a la persona mayor con enfermedades que generan dependencia, incluidas las crónico-degenerativas, las demencias y la enfermedad de Alzheimer. i) Fortalecer las capacidades de los trabajadores de los servicios de salud, sociales y socio-sanitarios integrados y de otros actores, en relación con la atención de la persona mayor, teniendo en consideración los principios contenidos en la presente Convención. j) Promover y fortalecer la investigación y la formación académica profesional y técnica especializada en geriatría, gerontología y cuidados paliativos. k) Formular, adecuar e implementar, según la legislación vigente en cada país, políticas referidas a la capacitación y aplicación de la medicina tradicional, alternativa y complementaria, en relación con la atención integral de la persona mayor. 1) Promover las medidas necesarias para que los servicios de cuidados paliativos estén disponibles y accesibles para la persona mayor, asi como para apoyar a sus familias. m) Garantizar a la persona mayor la disponibilidad y el acceso a los medicamentos reconocidos como esenciales por la Organización Mundial de la Salud, incluyendo los fiscalizados necesarios para los cuidados paliativos. n) Garantizar a la persona mayor el acceso a la información contenida en sus expedientes personales, sean físicos o digitales. o) Promover y garantizar progresivamente, y de acuerdo con sus capacidades, el acompañamiento y la capacitación a personas que ejerzan tareas de cuidado de la persona mayor, incluyendo familiares, con el fin de procurar su salud y bienestar.
} 
En este sentido el mencionado artículo establece también que los Estados Parte deberán diseñar medidas de: a) apoyo a las familias y cuidadores mediante la introducción de servicios para quienes realizan la actividad de cuidado de la persona mayor, teniendo en cuenta las necesidades de todas las familias y otras formas de cuidados, así como la plena participación de la persona mayor, respetándose su opinión, b) tendientes a desarrollar un sistema integral de cuidados que tenga especialmente en cuenta la perspectiva de género y el respeto a la dignidad e integridad física y mental de la persona mayor.

A esta enunciación de derechos agrega una serie garantías que aseguran su cumplimiento mediante el compromiso de los estados parte a:

“a) Establecer mecanismos para asegurar que el inicio y término de servicios de cuidado de largo plazo estén sujetos a la manifestación de la voluntad libre y expresa de la persona mayor.

b) Promover que dichos servicios cuenten con personal especializado que pueda ofrecer una atención adecuada e integral y prevenir acciones o prácticas que puedan producir daño o agravar la condición existente.

c) Establecer un marco regulatorio adecuado para el funcionamiento de los servicios de cuidado a largo plazo que permita evaluar y supervisar la situación de la persona mayor, incluyendo la adopción de medidas para:

i. Garantizar el acceso de la persona mayor a la información, en particular a sus expedientes personales, ya sean físicos o digitales, y promover el acceso a los distintos medios de comunicación e información, incluidas las redes sociales, así como informar a la persona mayor sobre sus derechos y sobre el marco jurídico y protocolos que rigen los servicios de cuidado a largo plazo.

ii. Prevenir injerencias arbitrarias o ilegales en su vida privada, familia, hogar o unidad doméstica, o cualquier otro ámbito en el que se desenvuelvan, así como en su correspondencia o cualquier otro tipo de comunicación.

iii. Promover la interacción familiar y social de la persona mayor, teniendo en cuenta a todas las familias y sus relaciones afectivas.

iv. Proteger la seguridad personal y el ejercicio de la libertad y movilidad de la persona mayor.

v. Proteger la integridad de la persona mayor y su privacidad e intimidad en las actividades que desarrolle, particularmente en los actos de higiene personal.

d) Establecer la legislación necesaria, conforme a los mecanismos nacionales, para que los responsables y el personal de servicios de cuidado a largo plazo respondan administrativa, civil y/o penalmente por los actos que practiquen en detrimento de la persona mayor, según corresponda. 
e) Adoptar medidas adecuadas, cuando corresponda, para que la persona mayor que se encuentre recibiendo servicios de cuidado a largo plazo cuente con servicios de cuidados paliativos que abarquen al paciente, su entorno y su familia".

Sin perjuicio de la operatividad de los tratados internacionales en nuestro derecho interno, mediante la publicación en el boletín oficial el 31 de mayo de 2017 se dispuso la aprobación de la Convención Interamericana sobre protección de los derechos humanos de las personas mayores, adoptada por la organización de los estados americanos durante la $45^{\mathrm{a}}$ asamblea general de la OEA.

En la provincia del Chaco se ha reconocido el derecho a la vejez a través de dos normas fundamentales en la materia: la Ley $\mathrm{N}^{\mathrm{O}} 7942$ sancionada el 14 de diciembre de 2016 y la Ley $\mathrm{N}^{\circ} 2761 \mathrm{G}$. Esta última ha adherido en todos sus artículos a lo dispuesto por la Ley Nacional 27.360, que aprueba la Convención Interamericana sobre Protección de los derechos humanos de las Personas Mayores, obligando en consecuencia a la provincia y a sus organismos a cumplir con los estándares que la convención fija.

Por otro lado, la Ley N ${ }^{0} 7942$ establece la promoción y protección integral de las personas adultas mayores. La mentada ley tiene por objeto garantizar el goce y ejercicio pleno de los derechos previstos en la constitución nacional, tratados internacionales, constitución provincial 1957-1994 en su art. 35 inc. 4 a fin de propiciar su integración plena al desarrollo social, económico, político y cultural de la provincia, contribuyendo al mejoramiento de su calidad de vida y su dignificación.

En nuestra legislación nacional tanto como la legislación internacional el derecho de la ancianidad estudia la condición jurídica de las personas que tengan sesenta o más años de edad considerando los sistemas de derechos, garantías y principios vinculados con el fenómeno demográfico del envejecimiento y el estado particular de cada persona que ha alcanzado la vejez.

Aún en ausencia de legislación específica nacional, la provincia del Chaco ha dado un paso adelante con esta ley que también se ha adelantado a reconocer el derecho de la comunidad a velar por el logro de la vigencia plena y efectiva de las prerrogativas y garantías de los adultos mayores. ${ }^{5}$

En su art. 7 efectúa una enumeración de principios rectores que deberán guiar la actuación institucional, pública y privada en todo lo concerniente a derechos de las personas Adultas Mayores. Consagra como principios generales la independencia, la participación, la atención prioritaria, la autorrealización y la dignidad.

El art. 26 por su parte, legisla específicamente en materia de residencias geriátricas o gerontológicas y dice:

"Se entiende por residencia gerontológica, a la institución de estadía permanente no sanatorial, destinada a la atención integral de adultos mayores autoválidos,

\footnotetext{
${ }^{5}$ Art. 4‥- Responsabilidades. Sin perjuicio de la responsabilidad primaria que le cabe al grupo familiar del adulto mayor y la que le es inherente a los organismos del Estado con competencia en la materia, la comunidad tiene el derecho, en el marco de una democracia participativa, de ser parte activa en el logro de la vigencia plena y efectiva de las prerrogativas y garantías de los adultos mayores, así como el deber de velar por la contención de los sujetos de la presente.
} 
o semidependientes para las actividades de la vida diaria, y cuya finalidad aspira a brindar asistencia nutricional, social, de confort, de rehabilitación, recreativas, culturales y cuidados médicos básicos, con especial énfasis en la prevención de factores de riesgo y la promoción de actividades que contribuyan a la calidad de vida".

Este reconocimiento aparece acompañado de la intangibilidad del presupuesto (art. 51) al efecto de que los fondos del presupuesto provincial destinados a la promoción y protección de derechos de las personas adultas mayores no pueda destinarse a fines distintos de los previstos. Destacamos que también dispone a renglón seguido que la previsión presupuestaria no puede ser en ningún caso inferior a la mayor previsión o ejecución de ejercicios anteriores.

Me honra decir que esto último se presenta en armonía con el principio de progresividad y no regresividad. La provincia, en materia legislativa, se ha mostrado a la altura de los estándares internacionales.

\section{DEFECTOS LEGISLATIVOS}

Fuera de las normas antes citadas, la demás legislación en la materia regula las residencias geriátricas por defecto. Encontramos un sinnúmero de leyes materiales y formales correspondientes a las obras sociales donde las residencias aparecen como prestadoras de servicios a las cuales se les exige el cumplimiento de lo que discrecionalmente las instituciones entienden como estándar mínimo para la cobertura de la prestación.

Ahora bien, esto plantea el problema del ejercicio de las facultades de contralor estatal. El estado no puede ejercer policía administrativa sobre actividades que no han sido previamente reguladas. La legislación por defecto deja librada la suerte de los adultos mayores a la legislación imperante en materias ajenas a las propias de las residencias geriátricas.

Por tanto, la suerte del adulto mayor se ve sometida a la obra social que le reconozca la prestación o a la mera exigencia que de modo particular pueda hacer respecto del servicio que le ofrecen. En particular, consideramos que la legislación imperante en la materia adolece de dos defectos en particular.

El primero de ellos es la categorización del adulto mayor según sea "Adulto Mayor" o "Adulto Mayor Psiquiátrico". De este modo lo establece el Programa de Atención Médica Integral -PAMI (INSJJ- Instituto Nacional de Servicios Sociales para Jubilados y Pensionados)- y consideramos que ambas categorías no se corresponden con la realidad de la persona mayor de 60 años ni con los paradigmas actuales de la geriatría. Creemos conveniente crear nuevas categorías que contemplen un lenguaje más inclusivo, que evalué las necesidades de la persona y no lo "categorice" según su salud psíquica. Las categorías que consideramos más adecuadas serían las de "adulto mayor semi dependiente" y "adulto mayor dependiente", contemplando 
ambas categorías la dependencia física y psíquica de terceros para llevar adelante actividades de la vida diaria.

A modo de propuesta, el grado de dependencia podría determinarse a través de índices utilizados nacionalmente, entre otros por PAMI, como el Índice de Katz, Índice de Tinetti e Índice de Barthel, que evalúan la independencia o dependencia que presenta el futuro residente para llevar adelante actividades de la vida diaria. Este diagnóstico podría ser efectuado por los auditores de los respectivos Institutos de Previsión Social (PAMI, en Nación o INSSSEP - Instituto de Seguridad Social, Seguros y Préstamos- en la Provincia del Chaco). Las residencias geriátricas, por su parte, podrían reservarse la facultad de presentar una reevaluación luego del primer mes de internación.

Es necesario además considerar que el adulto mayor puede no presentar las mismas necesidades en todo el transcurso de la internación. Por ello creemos que lo correcto sería que las auditorias se lleven adelante cada 6 meses para evaluar las capacidades del residente y en caso de haber aumentado o disminuido su dependencia, sea recategorizado. Esta categorización, lejos de ser una cuestión de vocabulario o meramente clasificatoria, presenta un impacto directo en los presupuestos que deben manejar las instituciones. Máxime si consideramos que los insumos tales como pañales, suplementos dietarios o medicación no serán los mismos para aquellos residentes que puedan valerse por sus propios medios respecto de aquellos que no puedan hacerlo. Una adecuada legislación, acorde a la realidad de cada persona, permite el acceso según la necesidad de cada residente.

En segundo lugar, creemos necesaria la adecuación de la cuantía monetaria de valor por prestación que paga la provincia del Chaco a los montos estipulados por PAMI a nivel nacional. Como hemos dicho antes, existe un interés público en la prestación del servicio de geriatría y, por lo tanto, debe existir una ley de contenidos mínimos nacional donde no podamos ir por debajo. La situación actual ha permitido la discriminación arbitraria de los adultos mayores. Actualmente, existen dos cánones o estándares dentro de la geriatría. Uno que corre por vía nacional y que económicamente permite al adulto mayor acceder a un nivel de prestaciones que otra parte de la población, tales como los adultos mayores cubiertos por las obras sociales de la provincia, se ven impedidos.

Una legislación especial en materia de residencias geriátricas, que establezca de manera específica el doble carácter de las contingencias que cubre, un piso mínimo de instalaciones y de personal por paciente, la unificación de los cánones mínimos ajustables conforme los aumentos salariales e inflacionarios, la previsión de las facultades de control del estado, son requisitos mínimos con los que debe contar cualquier persona en miras a un futuro digno.

El actual sistema normativo produce, desde nuestro punto de vista, una vulneración del anciano por no considerar su realidad específica, desprotegiéndolos ante las necesidades que las contingencias de la vejez y salud les provoca.

Desde el punto de vista de los valores jurídicos, hasta tanto la vejez no sea un factor determinante y no opere como un principio rector en la aplicación del derecho, no podremos brindar- 
le el efectivo acceso al anciano a los derechos de igualdad y vida digna que como ser humano le son propios. De manera concluyente entendemos que no podremos gozar con plenitud los derechos que nos son reconocidos sin que antes se nos reconozcan aquellos derechos más esenciales.

\section{CONCLUSIÓN}

Las Residencias Geriátricas son instituciones a disposición de las necesidades de alojamiento de la población que ya ha alcanzado la vejez. Son parte de una nueva construcción social más inclusiva que no se corresponde con las perspectivas legislativas que rigen la materia actualmente. La realidad del adulto mayor reclama atención sobre las contingencias de vejez y salud que los afecta. Solo es posible definir este enfoque legislativo desde una comprensión integral de la situación en la que se inserta.

La vejez debe ser considerada un factor determinante en la aplicación del derecho. Todo ello en miras a evitar la desregulación de situaciones que devienen en injustas y que, finalmente, desembocan en desprotección de una parte de la sociedad. La actual situación de desregulación coloca a los adultos mayores en un estado de vulnerabilidad. Por este motivo, necesitamos establecer un eje normativo que asegure a toda la población una vejez digna.

\section{REFERENCIAS BIBLIOGRAFÍCAS}

Alexy, R. (1993). Teoría de los derechos fundamentales (Garzón Valdés; E. trad.). Madrid: Centro de Estudios Constitucionales.

Alto Comisionado de las Naciones Unidas para los Refugiados (ACNUR). (2015). Principios de las Naciones Unidas a favor de las personas de edad. Recuperado el 12 de febrero de 2015; de http://www.acnur.org/t3/fileadmin/scripts/doc.php?file=t3/ fileadmin/Documentos/BDL/2003/1640; el 2 de marzo de 2015

Amaya, J. A. (2014). Control de constitucionalidad (2da. Edición). Buenos Aires-Bogotá: Astrea.

Amaya, J. A. (2015). Democracia y minoría política. Buenos Aires-Bogotá: Astrea.

Ansuategui Roig, F. J. De Asís Roig, R., Fernández García, E., Fernández Liesa, C. R. y Peces-Barba Martínez, G. (Dirs.). Historia de los derechos fundamentales. (pp. 29-o). Madrid: Dykinson. 
Arce y Flórez-Valdés, J. (1991). El derecho civil constitucional. Madrid: Civitas.

Ariza, A. (2003). Emergencia y sistema de derecho privado. Suplemento Lexis Nexis, 23 de abril.

Bazo, M. T. (1993). La vejez como problema social. En P. Sánchez Vera (ed.); Sociedad y Población Anciana. (pp. 181-193). Murcia: Universidad de Murcia.

Bellina Yrigoyen, J. (2004). La economía política de los fondos de pensiones. Rosario: Universidad del Centro Educativo Latinoamericano [UCEL].

Bernal Pulido, C. (2003). El principio de proporcionalidad y los derechos fundamentales. El principio de proporcionalidad como criterio par a determinar el contenido de los derechos fundamentales vinculante para el legislador. Madrid: Centro de Estudios Políticos y Constitucionales.

Bidart Campos, G. (1995). Tratado elemental de derecho Constitucional Argentino. (I), Buenos Aires: Ed. Ediar.

Bidart Campos, G. (1991). Teoría general de los derechos humanos. Buenos aires: Astrea.

Bobbio, N. (1991). El tiempo de los derechos (De Asís Roig; R. trad.). Madrid: Sistema.

Briones, C. (1998). La alteridad del cuarto mundo. Buenos Aires: Colihue.

Calvo Ortega, R. y García Calvente, Y. (2007). Situaciones de dependencia: regulación actual y nuevas perspectivas. Pamplona: Thomson; Civitas.

Chirinos, B. L. (2016). Derecho Previsional Argentino. (I), Buenos Aires: Ed. La Ley.

Ciuro Caldani, M. A. (1992). Derecho de la Ancianidad. Investigación y Docencia, (20, pp. 35-40).

Ciuro Caldani, M. A. (1995). Comparación jusfilosófica del Derecho de Menores y el Derecho de la Ancianidad. Investigación y Docencia. (25, pp.17-32).

Ciuro Caldani, M. Á. (2000). Metodología jurídica y lecciones de historia de la filosofía del derecho. Rosario: Fundación para las Investigaciones Jurídicas.

Ciuro Caldani, M. Á. (2000). La conjetura del funcionamiento de las normas jurídicas. Metodología jurídica. Rosario: Fundación para las Investigaciones Jurídica. 
Dabove, M. I. (2002). Los derechos de los ancianos (2da. Ed.). Buenos Aires: Ciudad Argentina.

Dabove, M. I. (2013b). Ciudadanía y derechos fundamentales de las personas mayores: de las políticas gerontológicas al Derecho de la Vejez. Revista de la Facultad de Derecho y Ciencias Sociales; 4, (I, pp. 19-36). Recuperado el 20 de mayo de 2014; de https://drive.google.com/ file/d/1PDno-knS5iwZwVaOLyf-dsP7SxPQimLh/view

Dabove, M. I. (2008). Derecho y multigeneracionismo: o los nuevos desafíos de la responsabilidad jurídica familiar en la vejez. Revista de Derecho de Familia; 40 (julio-agosto, pp. 39.54).

Dabove, M. I. (2013a). Elder law: a need that emerges in the course of life. Ageing International; 40 (septiembre, pp. 138-148). doi 10.1007/s12126-013-9193-4.

Dabove, M. I. y Di Tullio Budassi, R. G. (2009). Bases y puntos de partida para la convención internacional de derechos humanos en la ancianidad: la calidad de vida como meta. $X$ Congreso Nacional de Sociología Jurídica. Legalidad y Legitimidad: confrontaciones sociales en torno al Derecho. Córdoba; Consejo Latinoamericano de Ciencias Sociales -CLASCO-, Universidad Nacional de Rosario -UNC-; Sociedad Argentina de Sociología Jurídica.

Dabove, M. I. y Barbero, D. O. (2009). Igualdad y no discriminación en los actos de autoprotección: nuevas razones para la acción en favor de los derechos de los grupos vulnerables. Revista del Instituto de Derecho e Integración; (1, pp. 13-46).

Dabove, M. I. y Prunotto Laborde, A. (Dirs). (2006). Derecho de la Ancianidad. Perspectiva interdisciplinaria. Rosario: Juris.

Dayton, K. A. (Ed.). (2014). Comparative perspectives on adult guardianship. Durham: Carolina Academic Press.

Dayton, K. A. (2013). Elder law: readings; cases; and Materials. (4 ed.), Durham: Carolina Academic Press.

De Souza Minayo, M. C. (2006). Visâo antropológica do envelhecimento humano. Velhices. Reflexôes contemporáneas. San Pablo: Pontificia Universidad católica de Sao Paulo.

Di Tullio Budassi, R. (2008). El derecho alimentario de los ancianos. Derecho de Familia. Revista Interdisciplinaria de Doctrina y Jurisprudencia; (38, pp. 64-82). 
Duguit, L. (1927). Tratado Constitucional. (III). Ed. Fontemoing. Paris.

Etala, C. A. (2003). Infracciones en el derecho del trabajo. Buenos Aires: La Ley.

Fama, M. V. y Herrera, M. (2008). La obligación alimentaria de los abuelos hoy; Revista Jurídica online El Dial. 17 de noviembre (2659, pp. 34-52).

Frolik, L. A. y Kaplan, R. L. (2003). Elder law. (3 ed.) St. Paul: Thomson West.

Garcia Torres, J. y Jiménez-Blanco, A. (1986). Derechos fundamentales y relaciones entre particulares. Madrid: Civitas.

Geertz, C. (2006). Los usos de la diversidad. Barcelona: Paidós.

Goldin, A. (2009). Curso de Derecho del Trabajo y la Seguridad Social. Ed. La Ley. Buenos Aires.

Goldschmidt, W. (1987). Introducción filosófica al derecho. La teoría trialista del mundo jurídico y sus horizontes. (6 ed.) Buenos Aires: Depalma.

Herrera, M. (2011). Estado; ciudadanía y democracia. Algunos silenciados del derecho de familia: el papel de los abuelos en la familia del siglo XXI. Jurisprudencia Argentina. 16 de marzo (pp. 4-20).

Instituto Nacional de Estadísticas y Censos (INDEC). (2012). Encuesta Nacional sobre Calidad de Vida de Adultos Mayores 2012 - Encaviam; principales resultados. Serie Estudios Indec; (46, pp. 9-21).

Kymlica, W. (1995). Ciudadanía multicultural. Barcelona: Paidós.

Leeson, G. W. (2011). Prepared or not; Latin America faces the Challenge of aging; current history. Journal of Contemporary World Affairs. 110 (733, pp. 75-80).

Lesson, G. W. (2009a). Future ageing in southeast Asia: demographic trends; human capital and health status. En E. Nurvidya y A. Ananta (Eds.) (2009) Older Persons in Southeast Asia. (pp. 32-41). Singapore: Institute of Southeast Asian Studies -IEAS-.

Lesson, G. W. (2009b). Demography; politics and policy in Europe. En P. Ludow (Ed.); Setting EU Priorities 2009. Portugal: The European Strategy Forum. 
Mayer, O. (1954). Derecho administrativo alemán: Las obligaciones especiales: (servicio del Estado, cargas públicas, concesión de empresa pública, indemnizaciones por daños causados por la administración). Las personas morales, etc. Parte especial. Depalma.

Navarro, J. M. (2013). Policía del trabajo y administración laboral. Buenos Aires: Ed. La Ley.

Navarro, J. M. (2010). Policía del trabajo. Actuación de la administración pública en el ámbito de las relaciones de trabajo. Buenos Aires: Ed. Rubinzal Culzoni.

Nikken, P. (1986). Los derechos del niño; de los ancianos y de la mujer: su protección internacional. Revista del Instituto Interamericano de Derechos Humanos; (4, pp. 79-84).

Nino, C. S. (1990). Autonomía y necesidades básicas. Doxa; (7, pp. 73-79).

Nino, C. S. (2013). Fundamentos de derecho constitucional. (1ed.) Buenos Aires: Astrea.

Organización de las Naciones Unidas -ONU- (2002). Segunda Asamblea Mundial sobre el Envejecimiento Madrid, España. Plan de Acción Internacional de Madrid sobre el Envejecimiento 2002. Celebrada del 8 a 12 de abril. Recuperado de https://www.un.org/development/ desa/dspd/ [Consulta: 25 de junio de 2013].

Peces Barba Martínez, G. y Fernandez García, Eusebio (1998). Historia de los Derechos Fundamentales. Madrid: Dykinson.

Pérez Lu-o, A. E. (1995). Derechos humanos; estado de derecho y constitución. (5 ed.) Madrid: Tecnos.

Perez Lu-o, A. E. (1995). Derechos humanos; estado de derecho y constitución. (5 ed) Madrid: Tecnos.

Prieto Sanchis, L. (1990). Estudios sobre derechos fundamentales. Madrid: Debate.

Prieto Sanchis, L. (1992). Sobre principios y normas. Problemas del razonamiento jurídico. Madrid: Centro de Estudios Constitucionales.

Prieto Sanchis, L. (1994). Minorías; respeto a la disidencia e igualdad sustancial; en Doxa; (15-16, pp.367-387). 
Prieto Sanchís, L. (2002). Derechos fundamentales; neoconstitucionalismo y ponderación judicial. Lima: Palestra.

Rosario, Z. (2007). Estrategia jurídica. Rosario: UNR Editora.

Sagûes, N. P. (2014). Manual de derecho constitucional (2 ed.) Buenos Aires: Astrea.

Sartori, G. (2001). La sociedad multiétnica. Pluralismo; multiculturalismo y extranjeros. Madrid: Taurus.

Sen, A. (2011). La idea de justicia (Valencia Villa; H. trad.). Buenos Aires: Aguilar, Altea.

Taylor, C. (1993). Multiculturalismo y la política del reconocimiento. México; D. F.: Fondo de Cultura Económica.

United Nation. (2015). Open-ended Working Group on Ageing for the purpose of strengthening the protection of the human rights of older persons. Fecha de consulta: 02/03/15. Disponible en: http://social.un.org/ageing-working-group/

Vázquez Vilard, A. y Navarro, M. J. (1990). Policía del trabajo. Buenos Aires: Ed. Astrea.

Yturbe, C. de (2008). Multiculturalismoy derechos. México; D.F.: Instituto Federal Electoral. Fecha de consulta: 05/06/13. Disponible en: http://biblio.juridicas.unam.mx/libros/1/490/1.pdf http://dx.doi.org/10.14198/DOXA1990.7.01

\section{CURRICULUM VITAE}

\section{Ayelén Anzola}

Abogada por la Universidad Nacional del Nordeste, Facultad de Derecho y Ciencias Sociales y Políticas. Especialista en Seguridad Social por la Universidad Nacional del Nordeste, Facultad de Derecho y Ciencias Sociales y Políticas Maestranda en Derecho Administrativo por la Universidad Austral - (estado: confección de tesis magistral). Maestranda en Estrategias Anticorrupción y Políticas de Integridad por la Universidad de Salamanca - España. Auxiliar docente de Primera - dedicación simple - Universidad Nacional del Nordeste - Facultad de Ciencias Económicas - Asignatura: Instituciones de Derecho Público. Docente Investigador. Universidad Nacional del Nordeste - Facultad de Ciencias Económicas ayeanzola@hotmail.com 